http://dx.doi.org/10.35381/r.k.v6i11.1087

\title{
Satisfacción Laboral en colaboradores de una cadena mexicana de supermercados
}

\section{Job Satisfaction in collaborators of a mexican chain of supermarkets}

\author{
Isabel Solís-Magaña \\ isabelsolismagana5434@gmail.com \\ Universidad Juárez Autónoma de Tabasco, Tabasco \\ México \\ https://orcid.org/0000-0002-4171-112X \\ Fabiola de Jesús Mapén-Franco \\ mapenfranco@hotmail.com \\ Universidad Juárez Autónoma de Tabasco, Tabasco \\ México \\ https://orcid.org/0000-0002-8436-4615 \\ Wilver Méndez-Magaña \\ mendezwilver@yahoo.com \\ Universidad Juárez Autónoma de Tabasco, Tabasco \\ México \\ https://orcid.org/0000-0003-2975-1505
}

Recepción: 10 de julio 2020

Revisado: 29 de agosto 2020

Aprobación: 17 de diciembre 2020

Publicación: 01 de enero 2021 


\title{
RESUMEN
}

La presente investigación tuvo como propósito principal determinar el nivel de satisfacción de los colaboradores de una cadena de supermercados ubicada en el estado de Tabasco, México. El diseño metodológico de la investigación fue transversal descriptivo, no experimental, con enfoque cuantitativo, aplicando el instrumento de Meliá y Peiró (1998) adaptado por expertos en el área del comportamiento organizacional y validado a través del Alfa de Cronbach con 0.97. Los resultados reflejan una presencia alta de satisfacción en las dimensiones evaluadas a través del cuestionario, el nivel de satisfacción se relaciona con los años de antigüedad en la empresa de manera positiva.

Descriptores: Satisfacción en el trabajo; motivación; actitud laboral. (Palabras tomadas del Tesauro UNESCO).

\begin{abstract}
The main purpose of this research was to determine the satisfaction level of the collaborators of a supermarket chain located in the state of Tabasco, Mexico. The methodological design of the research was descriptive, non-experimental,cross-sectional, with a quantitative approach, applying the Meliá y Peiró (1998) instrument adapted by experts in the area of organizational behavior and validated through Cronbach's Alpha with 0.97 . The results reflect a high presence of satisfaction in the dimensions evaluated through the questionnaire, the level of satisfaction is related to the years of seniority in the company in a positive way.
\end{abstract}

Descriptors: Job satisfaction; motivation; work attitude. (Words taken from the UNESCO Thesaurus). 


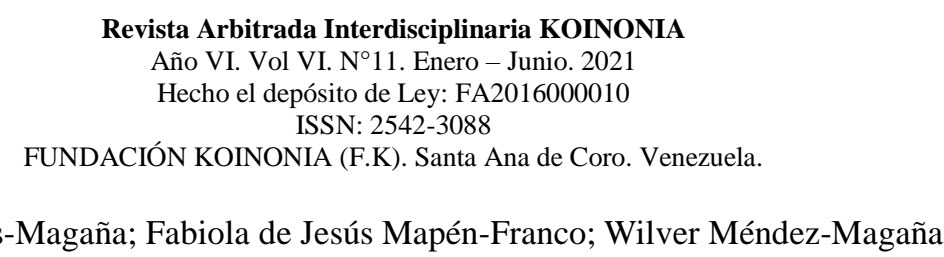

Isabel Solís-Magaña; Fabiola de Jesús Mapén-Franco; Wilver Méndez-Magaña

\section{INTRODUCCIÓN}

Las organizaciones han implementado diversas estrategias para alcanzar sus objetivos y metas, una de ellas es medir la satisfacción laboral ya que ésta repercute directamente en el ambiente que perciben los trabajadores. La relevancia de este constructo teórico se comprende desde los ámbitos de la Psicología Organizacional que reconoce el efecto mediador que permea entre las condiciones sociolaborales y el desarrollo de las organizaciones (Mapén, 2020). Adrián, Buboltz, Walter y Winkelspecht (2004) afirman que la satisfacción laboral influye en los resultados de los trabajadores. Chiang, Méndez y Sánchez (2010) señalan a la década de los 30, en la cual se originó un gran interés por el concepto de satisfacción en el trabajo, alcanzando su punto máximo en los años 60 , pero es hasta los años 80 cuando se comienza a relacionar con la calidad de vida que se genera en el trabajo, el impacto que genera en la salud mental y la familia.

En la actualidad diversas investigaciones han correlacionado la satisfacción laboral positivamente con diversas variables como: el desempeño laboral, el clima organizacional, el rendimiento, productividad o compromiso organizacional (Mañas, Salvador, Boada, González y Agulló 2007; Rodríguez, Paz, Lizana y Cornejo 2011; Chiang y San Martín 2015; Herrera y Sánchez 2012; Juárez y Salvador 2012). De igual manera otros estudios han identificado las diferencias que se perciben de la satisfacción laboral influidas por diversas características sociodemográficas (Pilar 2008; Tejero y Fernández, 2009).

Actualmente se ha declarado la importancia que tiene el estado psicológico de las personas en los diferentes tipos de organización, ya que éste puede ser un elemento determinante para el desempeño del trabajador (Ramírez y Mapén, 2019). Al respecto, la satisfacción laboral se ha convertido en un tema de interés para cualquier organización que busca conocer los múltiples factores que influyen en el bienestar psicológico de los trabajadores (Sánchez y García, 2017).

Al respecto la presente investigación persigue como objetivo central identificar y describir el nivel de satisfacción laboral presente en los empleados del área de caja de los supermercados de una cadena mexicana ubicados en el estado de Tabasco, México. La 


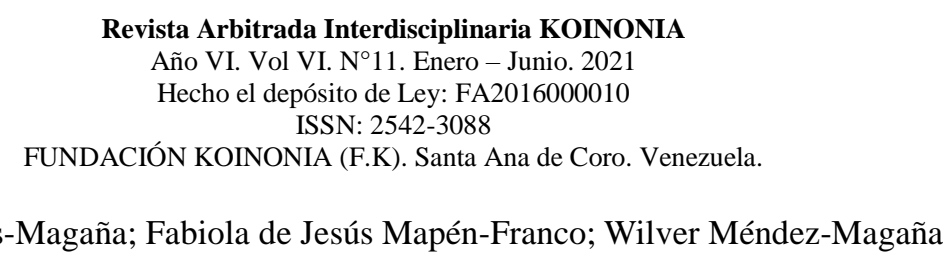

Isabel Solís-Magaña; Fabiola de Jesús Mapén-Franco; Wilver Méndez-Magaña

importancia del estudio se comprende a partir de las funciones de los empleados del área de caja ya que son ellos quienes están en constante interacción con los clientes. Por lo que a partir de conocer los niveles de satisfacción laboral, la organización podrá tomar decisiones al conocer la percepción de los trabajadores de las condiciones de trabajo y que en consecuencia inciden en su comportamiento y desempeño.

\section{Revisión Teórica y Operacionalización de la Variable: Satisfacción Laboral}

La satisfacción laboral es un tema muy estudiado en el ámbito de las grandes y medianas empresas, Atalaya (1999) señala que entender el comportamiento organizacional toma cada vez más relevancia debido a los cambios que surgen en el entorno y que pueden representar retos o bien oportunidades dentro de las organizaciones. De igual manera manifiesta que con base en las teorías humanistas los trabajadores más satisfechos son aquellos que logran cubrir sus necesidades psicológicas y sociales en sus labores, lo que generalmente es relacionado con resultados positivos.

Blanco (2015) afirma que la satisfacción laboral es una variable dependiente del comportamiento organizacional y de las condiciones laborales en las que se encuentre el trabajador dentro de la organización. Mientras tanto Sánchez y García (2017) plantean que el éxito de las organizaciones modernas se debe a la integración de los trabajadores para perseguir los objetivos de la misma organización como propios.

Davis y Newstrom, (1999) citado en Rodríguez, Paz, Lizana y Cornejo (2011) mencionan que la satisfacción laboral ha sido definida de múltiples formas, considerándose al principio como un estado emocional o afectivo de los individuos hacia su trabajo. De igual manera Salgado, Remeseiro e Iglesias (1996) citando a (Locke, 1976, 1984) definen a la satisfacción "como una respuesta emocional positiva al puesto y que resulta de la evaluación de si el puesto cumple o permite cumplir los valores laborales del individuo" (p. 330). Mientras tanto Chiang, Méndez y Sánchez (2010) citando a (Werther y Davis, 1982) le proporcionan otro sentido, afirmando "es como el conjunto de sentimientos favorables y desfavorables mediante los cuales los trabajadores perciben su empleo" ( $p$. 22). Sin embargo, consideran que lo esencial es reconocer que la satisfacción es un 


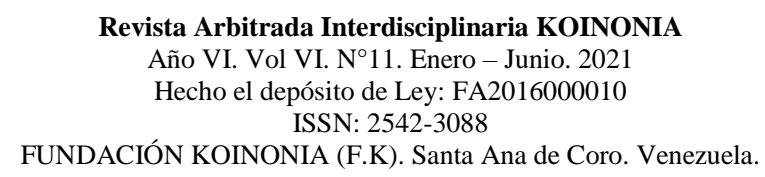

Isabel Solís-Magaña; Fabiola de Jesús Mapén-Franco; Wilver Méndez-Magaña

sentimiento de relativo placer o dolor, lo que se diferencia de los pensamientos objetivos y del comportamiento, a pesar de ser tres factores que ayudan a la dirección de la empresa, a comprender la reacción de los trabajadores ante su empleo.

Pilar (2008) da a conocer que un grupo de investigadores en los que se destaca (Crites, 1969; Locke, 1976; Smith, Kendall y Hullin, 1969) hacen referencia a la satisfacción laboral como un estado emocional, sin embargo otro grupo (Arnold, Robertson y Cooper, 1991; Beer, 1964; Bravo, 1992; Griffin y Bateman, 1986; Harpaz, 1983; Peiró, 1984; Salancik y Pfeffer, 1977) lo caracterizan como una actitud generalizada ante el trabajo, y concluye que en la mayoría de las aportaciones se afirma que "tienen que ver con la satisfacción con el contenido del trabajo (interés intrínseco del trabajo, la variedad, las oportunidades de aprendizaje o la dificultad), con el salario, con las condiciones de trabajo (horario, descansos, condiciones ambientales) y con la empresa." (p. 27). Boluarte (2014) señala que para la organización es importante conocer estas actitudes de su personal, con la finalidad de prevenir malas situaciones y mejorar la satisfacción de su personal. Para Mañas, Salvador, Boada, González y Agulló (2007) "la satisfacción laboral es una variable actitudinal que parece afectar al compromiso, a su vez, ésta se encuentra estrechamente vinculada con otras variables psicológicas (bienestar psicológico y satisfacción con la vida)" (p.396).

De acuerdo con Rodríguez, Paz, Lizana y Cornejo (2011) aluden a la satisfacción laboral como un constructo que existe en consenso con el clima y desempeño laboral, siendo la satisfacción laboral un predictor de rendimiento y productividad, las cuales son fundamentales para la gestión de las organizaciones. Al respecto, Chiang y San Martín (2015) realizaron un estudio de la satisfacción laboral y el desempeño laboral, encontrando una correlación estadísticamente significativa, cuando se aumenta la productividad, aumenta su satisfacción respecto a su ambiente físico, desarrollo y mejora el ambiente.

Para Pilar (2008) determinar la satisfacción laboral es de suma importancia en las organizaciones ya que permite conocer la habilidad de la organización para satisfacer las necesidades de los empleados; además se ha evidenciado que los trabajadores 
Revista Arbitrada Interdisciplinaria KOINONIA

Año VI. Vol VI. Nº11. Enero - Junio. 2021

Hecho el depósito de Ley: FA2016000010

ISSN: 2542-3088

FUNDACIÓN KOINONIA (F.K). Santa Ana de Coro. Venezuela.

Isabel Solís-Magaña; Fabiola de Jesús Mapén-Franco; Wilver Méndez-Magaña

insatisfechos faltan a trabajar con más frecuencia y suelen renunciar a sus trabajos, mientras que los empleados satisfechos permanecen más tiempo en la organización y tienen un mejor desempeño.

Meliá y Peiró (1998) evalúan la satisfacción laboral (ver tabla 1) mediante un instrumento tomando en cuenta las restricciones motivacionales y temporales a los que un trabajador puede estar expuesto, midiendo cinco factores.

Tabla 1.

Operacionalización de la Variable: Satisfacción Laboral.

\begin{tabular}{|c|c|c|c|c|c|c|}
\hline Variable & Autores & Concepto & Dimensiones & Autor & Concepto & Ítem \\
\hline $\begin{array}{c}\text { Satisfacción } \\
\text { Laboral }\end{array}$ & $\begin{array}{c}\text { (Chiang, } \\
\text { Méndez y } \\
\text { Sánchez 2010, } \\
\text { Mañas, } \\
\text { Salvador, } \\
\text { Boada, } \\
\text { González y } \\
\text { Agulló 2007, } \\
\text { Rodríguez, Paz, } \\
\text { Lizana y } \\
\text { Cornejo 2011, } \\
\text { Chiang y San } \\
\text { Martín 2015, } \\
\text { Salgado, } \\
\text { Remeseiro y } \\
\text { Iglesias 1996, } \\
\text { Pilar 2008). }\end{array}$ & $\begin{array}{c}\text { Es un estado } \\
\text { emocional o } \\
\text { afectivo que } \\
\text { perciben los } \\
\text { trabajadores } \\
\text { ante la } \\
\text { organización } \\
\text { en } \\
\text { consecuencia } \\
\text { con el } \\
\text { contenido del } \\
\text { trabajo. }\end{array}$ & $\begin{array}{l}\text { Satisfacción con } \\
\text { el ambiente } \\
\text { físico de Trabajo }\end{array}$ & $\begin{array}{c}\text { ( Meliá y } \\
\text { Peiró 1998) }\end{array}$ & $\begin{array}{c}\text { Forma en que los } \\
\text { superiores juzgan la } \\
\text { tarea, la supervisión } \\
\text { recibida, la } \\
\text { proximidad, frecuencia } \\
\text { de supervisión, el } \\
\text { apoyo recibido de los } \\
\text { supervisores y a la } \\
\text { igualdad y justicia de } \\
\text { trato recibida de la } \\
\text { empresa. }\end{array}$ & $\begin{array}{l}\text { 13.- Las relaciones personales } \\
\text { con sus superiores, 14.- La } \\
\text { supervisión que ejercen sobre } \\
\text { usted, 15.- La proximidad y } \\
\text { frecuencia con que es } \\
\text { supervisado, 16.- La forma en } \\
\text { que sus supervisores juzgan } \\
\text { su } \\
\text { tarea, 17.- La "igualdad" y } \\
\text { "justicia" de trato que recibe } \\
\text { de su empresa, y 18.- El apoyo } \\
\text { que recibe de sus superiores. } \\
\text { 6.- La limpieza, higiene y } \\
\text { salubridad de su lugar de } \\
\text { trabajo, } 7 .- \text { El entorno físico y } \\
\text { el espacio de que dispone en } \\
\text { su lugar de trabajo, } 8 .- \text { La } \\
\text { iluminación de su lugar de } \\
\text { trabajo, 9.- La ventilación de } \\
\text { su lugar de trabajo y } 10 .- \text { La } \\
\text { temperatura de su local de } \\
\text { trabajo. } \\
\text { 4.- El salario que usted } \\
\text { recibe, } 11 .- \text { Las } \\
\text { oportunidades de formación } \\
\text { que le ofrece la empresa, } \\
\text { 12.- Las oportunidades de } \\
\text { promoción que tiene, } 22 .- \text { El } \\
\text { grado en que su empresa } \\
\text { cumple el convenio, } \\
\text { las disposiciones y leyes } \\
\text { laborales y } 23 .- \text { La forma en } \\
\text { que se da la negociación en } \\
\text { su } \\
\text { empresa sobre aspectos } \\
\text { laborales. }\end{array}$ \\
\hline
\end{tabular}




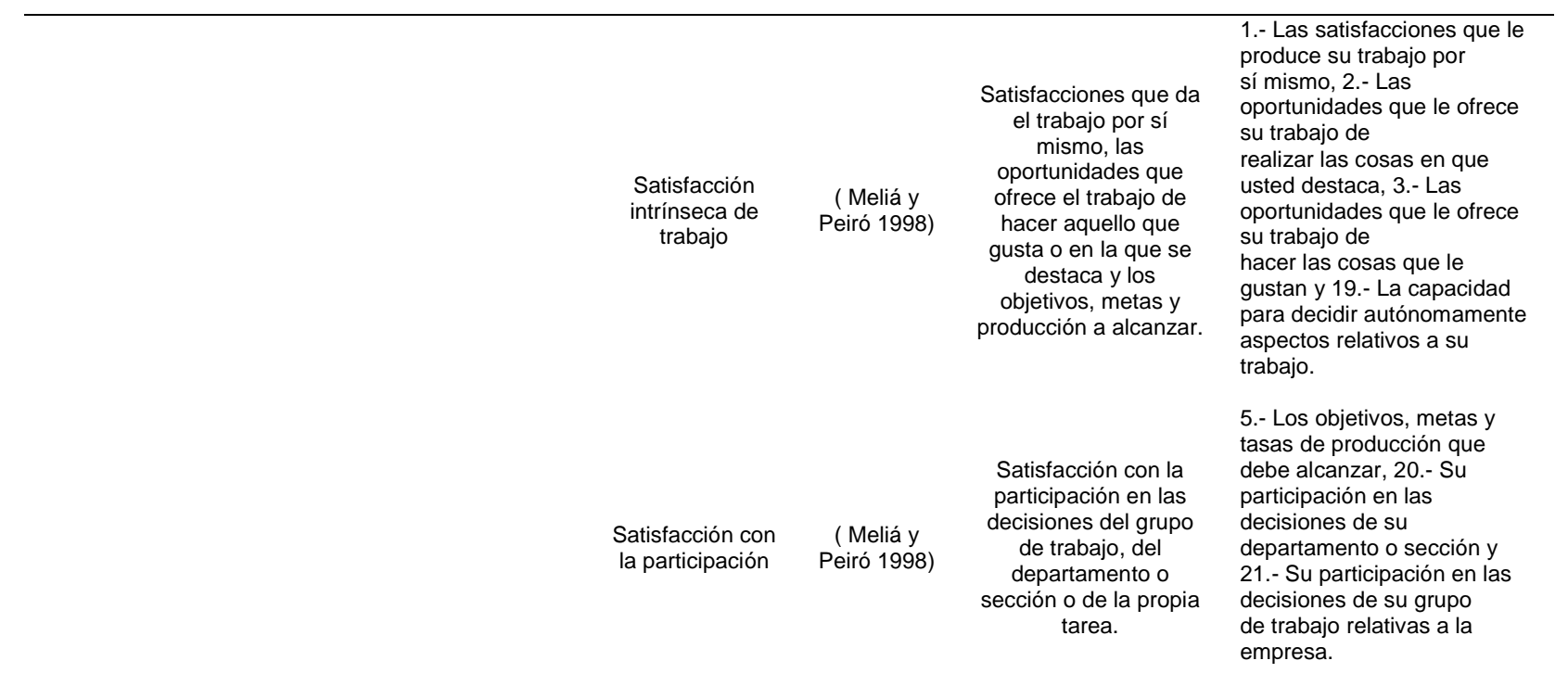

Fuente: Elaboración propia con base a diversos autores.

\section{MÉTODO}

El método de la investigación es descriptivo ya que el propósito fue determinar el nivel de satisfacción de los colaboradores hacia la empresa, con un diseño no experimental, debido a que no se manipuló ninguna variable, es decir, los colaboradores tienen su propia perspectiva de la empresa. El enfoque es cuantitativo, debido a que se utilizó el cuestionario aceptado y validado de los autores Meliá y Peiró (1998), adaptando su contenido a los requerimientos y contexto de la empresa objeto de estudio, a través del juicio de expertos, en el cual se consideró la experiencia en investigaciones y disciplina de Doctores en Estudios Organizacionales y verificando su fiabilidad a través del Alpha de Cronbach con resultado igual a 0.97 , por lo que la fiabilidad es aceptable (Hernández, Fernández y Baptista, 2014).

El instrumento de Meliá y Peiró (1998) denominado Cuestionario de Satisfacción Laboral S20/23, consta de 23 ítems, realizando una adecuación de 7 puntos que utilizan los autores a sólo 5 puntos de escala tipo Likert, en donde 1 es muy insatisfecho, 2 es insatisfecho, 3 indiferente, 4 satisfecho y 5 muy satisfecho, con una dirección favorable como se puede observar. Este cuestionario está integrado en cinco dimensiones de 
análisis: satisfacción con la supervisión ( 6 ítems), satisfacción con el ambiente (5 ítems), prestaciones percibidas ( 5 ítems), satisfacción intrínseca de trabajo (4 ítems), satisfacción con la participación (3 ítems). Además de solicitar características sociodemográficas, antigüedad laboral y escolaridad alcanzada

La población de estudio fueron colaboradores de las sucursales de una cadena mexicana ubicadas en la ciudad de Villahermosa, capital de estado de Tabasco en México. La totalidad fue de 42 empleados del área de caja, donde 25 corresponden a una sucursal y 17 empleados a otra. A continuación (Tabla 2) se describen las características sociodemográficas de los empleados en ambos supermercados.

\section{Tabla 2.}

Características sociodemográficas de los empleados encuestados.

\begin{tabular}{cccc}
\hline & Datos & Valores & $\%$ \\
\hline \multirow{2}{*}{ Género } & Masculino & 16 & $38 \%$ \\
& Femenino & 26 & $62 \%$ \\
& Casado & 9 & $22 \%$ \\
Estado Civil & Soltero & 22 & $52 \%$ \\
& Divorciado & 6 & $14 \%$ \\
& Unión Libre & 5 & $12 \%$ \\
& & & \\
Antigüedad & Menos de 1 año & 7 & $17 \%$ \\
Laboral & 1 año & 11 & $26 \%$ \\
& 2 años & 10 & $24 \%$ \\
& 3 años & 8 & $19 \%$ \\
Grado máximo & 4 años & 6 & $14 \%$ \\
de Estudios & & & $5 \%$ \\
& Secundaria & 2 & $12 \%$ \\
\hline
\end{tabular}

Fuente: Elaboración propia. 


\section{RESULTADOS}

En este apartado se presenta el análisis estadístico a partir de las cinco dimensiones que evalúan la satisfacción que integran el instrumento de los autores Meliá y Peiró (1998), teniendo en cuenta como el máximo y mínimo puntaje que los encuestados pudieran obtener se clasificó en los niveles que se presentan a continuación.

\section{Tabla 3.}

Puntaje para la clasificación del nivel de satisfacción.

\begin{tabular}{ll}
\hline Puntaje & Nivel de Satisfacción \\
\hline $23-41$ & Muy insatisfecho \\
$42-60$ & Insatisfecho \\
$61-79$ & Indiferente \\
$80-97$ & Satisfecho \\
$98-115$ & Muy satisfecho \\
\hline
\end{tabular}

\section{Fuente: Elaboración propia.}

Se realizó un recuento de los niveles obtenidos en las encuestas realizadas al personal por cada factor, a continuación, se analiza cada factor (Tabla 4); en la primera dimensión que mide la satisfacción que tiene el personal con la supervisión, se observa una mayor concentración en un nivel satisfecho $57 \%$, por lo que existe una tendencia positiva hacia una buena satisfacción. En la segunda dimensión, la satisfacción que tienen los empleados con su ambiente físico de trabajo, tiene un mayor recuento en un nivel satisfecho $43 \%$ seguida de un $21 \%$ que se encuentra indiferente y el $17 \%$ muy satisfecho. Para las demás dimensiones se presenta la misma tendencia, encuentran el mayor recuento en el nivel satisfecho: satisfacción con las prestaciones percibidas 40\%, satisfacción intrínseca de trabajo $40 \%$ y la satisfacción con la participación $48 \%$, las cinco dimensiones medidas tienen una concentración mayor a los ítems positivos, lo que puede inferirse en que sí existe satisfacción. 


\section{Tabla 4.}

Distribución de las dimensiones de la satisfacción laboral.

\begin{tabular}{|c|c|c|c|c|c|c|c|c|c|c|}
\hline \multirow[t]{2}{*}{ Nivel } & \multicolumn{2}{|c|}{$\begin{array}{l}\text { Satisfacción } \\
\text { con la } \\
\text { supervisión }\end{array}$} & \multicolumn{2}{|c|}{$\begin{array}{c}\text { Satisfacción } \\
\text { con el } \\
\text { ambiente físico } \\
\text { de trabajo }\end{array}$} & \multicolumn{2}{|c|}{$\begin{array}{c}\text { Prestaciones } \\
\text { percibidas }\end{array}$} & \multicolumn{2}{|c|}{$\begin{array}{l}\text { Satisfacción } \\
\text { intrínseca de } \\
\text { trabajo }\end{array}$} & \multicolumn{2}{|c|}{$\begin{array}{l}\text { Satisfacción } \\
\text { con la } \\
\text { participación }\end{array}$} \\
\hline & Recuento & $\%$ & Recuento & $\%$ & Recuento & $\%$ & Recuento & $\%$ & Recuento & $\%$ \\
\hline insatisfecho & 4 & $10 \%$ & 3 & $7 \%$ & 6 & $14 \%$ & 3 & $7 \%$ & 4 & $10 \%$ \\
\hline Insatisfecho & 2 & $5 \%$ & 5 & $12 \%$ & 2 & $5 \%$ & 7 & $17 \%$ & 3 & $7 \%$ \\
\hline Indiferente & 6 & $14 \%$ & 9 & $21 \%$ & 8 & $19 \%$ & 6 & $14 \%$ & 11 & $26 \%$ \\
\hline Satisfecho & 24 & $57 \%$ & 18 & $43 \%$ & 17 & $40 \%$ & 17 & $40 \%$ & 20 & $48 \%$ \\
\hline $\begin{array}{c}\text { Muy } \\
\text { satisfecho }\end{array}$ & 6 & $14 \%$ & 7 & $17 \%$ & 9 & $21 \%$ & 9 & $21 \%$ & 4 & $10 \%$ \\
\hline Total & 42 & 100 & 42 & 100 & 42 & 100 & 42 & 100 & 42 & 100 \\
\hline
\end{tabular}

Fuente: Elaboración propia.

A continuación, se describe el nivel de satisfacción laboral considerando cada una de las características sociodemográficas: género, estado civil, edad, antigüedad laboral y grado de estudio. Para la característica del género, las mujeres obtuvieron un $58 \%$ en un nivel satisfecho, seguido de un $23 \%$ indiferente, los hombres un $56 \%$ en un nivel satisfecho y un 19\% que se encuentra indiferente, ambos géneros siguen el mismo patrón de distribución, por lo que se puede indicar que el género no es un factor determinante en el nivel de satisfacción de los colaboradores. 


\section{Tabla 5.}

Distribución de los niveles de satisfacción por género.

\begin{tabular}{ccccc}
\hline Nivel & \multicolumn{2}{c}{ Masculino } & \multicolumn{2}{c}{ Femenino } \\
\hline $\begin{array}{c}\text { Muy } \\
\text { insatisfecho }\end{array}$ & 2 & $13 \%$ & 2 & $8 \%$ \\
$\begin{array}{c}\text { Insatisfecho } \\
\text { Indiferente }\end{array}$ & 1 & $6 \%$ & 2 & $8 \%$ \\
Satisfecho & 3 & $19 \%$ & 6 & $23 \%$ \\
$\begin{array}{c}\text { Muy } \\
\text { satisfecho }\end{array}$ & 9 & $56 \%$ & 15 & $58 \%$ \\
Total & 1 & $6 \%$ & 1 & $4 \%$ \\
\hline
\end{tabular}

\section{Fuente: Elaboración propia}

Para la evaluación del estado civil se consideraron cuatro estados, los casados con un $56 \%$ en el nivel satisfecho y con un $22 \%$ que se encuentran indiferentes e insatisfecho, sin presencia en ambos extremos de los niveles de satisfacción. Para los solteros un $59 \%$ en el nivel satisfecho, seguido de un $27 \%$ de trabajadores indiferentes. Los trabajadores divorciados tienen el $50 \%$ en el nivel satisfecho. Para el último estado civil (unión libre) se obtuvo un $60 \%$ en el nivel satisfecho, así se puede observar que en todos los casos se tiene mayor frecuencia en un nivel satisfecho, por lo que se considera que el estado civil no incide en los niveles de satisfacción. 


\section{Tabla 6.}

Distribución de los niveles de satisfacción por estado civil.

\begin{tabular}{ccccccccc}
\hline Nivel & \multicolumn{2}{c}{ Casado } & \multicolumn{2}{c}{ Soltero } & \multicolumn{2}{c}{ Divorciado } & \multicolumn{2}{c}{ Unión Libre } \\
\hline $\begin{array}{c}\text { Muy } \\
\text { insatisfecho }\end{array}$ & Recuento & $\%$ & Recuento & $\%$ & Recuento & $\%$ & Recuento & $\%$ \\
Insatisfecho & 2 & $22 \%$ & 0 & & 0 & & 1 & $20 \%$ \\
Indiferente & 2 & $22 \%$ & 6 & $27 \%$ & 1 & $17 \%$ & 0 & \\
Satisfecho & 5 & $56 \%$ & 13 & $59 \%$ & 3 & $50 \%$ & 3 & $60 \%$ \\
$\quad \begin{array}{c}\text { Muy } \\
\text { satisfecho }\end{array}$ & 0 & $0 \%$ & 1 & $5 \%$ & 1 & $17 \%$ & 0 & \\
Total & $\mathbf{9}$ & $\mathbf{1 0 0}$ & $\mathbf{2 2}$ & $\mathbf{1 0 0}$ & $\mathbf{6}$ & $\mathbf{1 0 0 \%}$ & $\mathbf{5}$ & $\mathbf{1 0 0 \%}$ \\
\hline
\end{tabular}

Fuente: Elaboración propia.

La distribución por niveles de satisfacción por años de antigüedad en la personal (Tabla 7) muestra que los colaboradores que solo tienen meses en la empresa $71 \%$ de ellos se sienten satisfechos, siendo este el porcentaje más alto presente. Seguido de un $70 \%$ de nivel satisfecho correspondiente a los empleados con dos años de antigüedad laboral. Sucesivo por el $67 \%$ de empleados con cuatro años de antigüedad satisfechos, y con un $64 \%$ en el mismo nivel satisfecho los empleados con un año en la empresa. Aunque todas las demás antigüedades siguen su mayor concentración en el nivel de empleados satisfechos, se nota una distribución diferente en los empleados de tres años de antigüedad, sintiéndose insatisfechos e indiferentes en un $38 \%$ cada uno. 
Tabla 7.

Distribución de los niveles de satisfacción por año de antigüedad.

\begin{tabular}{|c|c|c|c|c|c|c|c|c|c|c|}
\hline \multirow[t]{2}{*}{ Nivel } & \multicolumn{2}{|c|}{ Meses } & \multicolumn{2}{|c|}{1 año } & \multicolumn{2}{|c|}{2 años } & \multicolumn{2}{|c|}{3 años } & \multicolumn{2}{|c|}{4 años } \\
\hline & Recuento & $\%$ & Recuento & $\%$ & Recuento & $\%$ & Recuento & $\%$ & Recuento & $\%$ \\
\hline $\begin{array}{c}\text { Muy } \\
\text { insatisfecho }\end{array}$ & 2 & $29 \%$ & 2 & $18 \%$ & 0 & & 0 & & 0 & \\
\hline Insatisfecho & 0 & & 0 & & 0 & & 3 & $38 \%$ & 0 & \\
\hline Indiferente & 0 & & 2 & $18 \%$ & 2 & $20 \%$ & 3 & $38 \%$ & 2 & $33 \%$ \\
\hline Satisfecho & 5 & $71 \%$ & 7 & $64 \%$ & 7 & $70 \%$ & 1 & $12 \%$ & 4 & $67 \%$ \\
\hline $\begin{array}{c}\text { Muy } \\
\text { satisfecho }\end{array}$ & 0 & & 0 & & 1 & $10 \%$ & 1 & $12 \%$ & 0 & \\
\hline Total & 7 & $100 \%$ & 11 & $100 \%$ & 10 & $100 \%$ & 8 & $100 \%$ & 6 & $100 \%$ \\
\hline
\end{tabular}

Fuente: Elaboración propia.

Para conocer la distribución de los niveles de satisfacción por escolaridad de los colaboradores se consideró la educación media a la educación superior, y se distribuye de la siguiente manera (Tabla 8), los empleados con estudios concluidos de secundaria se distribuye equitativamente entre $50 \%$ se encuentran indiferentes y $50 \%$ se sienten satisfechos, para los empleados con la educación hasta preparatoria y licenciatura se concentra el mayor porcentaje en el nivel de empleados satisfechos con un $57 \%$ y $60 \%$ respectivamente. 


\section{Tabla 8.}

Distribución de los niveles de satisfacción por escolaridad alcanzada.

\begin{tabular}{|c|c|c|c|c|c|c|}
\hline \multirow{3}{*}{$\begin{array}{c}\text { Muy } \\
\text { insatisfecho }\end{array}$} & \multicolumn{2}{|c|}{ Secundaria } & \multicolumn{2}{|c|}{ Preparatoria } & \multicolumn{2}{|c|}{ Licenciatura } \\
\hline & Recuento & $\%$ & Recuento & $\%$ & Recuento & $\%$ \\
\hline & 0 & & 4 & $11 \%$ & 0 & \\
\hline Insatisfecho & 0 & & 3 & $9 \%$ & 0 & \\
\hline Indiferente & 1 & $50 \%$ & 6 & $17 \%$ & 2 & $40 \%$ \\
\hline Satisfecho & 1 & $50 \%$ & 20 & $57 \%$ & 3 & $60 \%$ \\
\hline $\begin{array}{c}\text { Muy } \\
\text { satisfecho }\end{array}$ & 0 & 0 & 2 & $6 \%$ & 0 & \\
\hline Total & 2 & 100 & 35 & 100 & 5 & $100 \%$ \\
\hline
\end{tabular}

Fuente: Elaboración propia.

\section{CONCLUSIONES}

Los resultados obtenidos dan pauta para confirmar que, si existe una relación de satisfacción con los factores evaluados en el instrumento, es decir, la satisfacción con la supervisión, la satisfacción con el ambiente físico de trabajo, las prestaciones percibidas, la satisfacción intrínseca de trabajo y la satisfacción con la participación, tienen altos niveles en su mayoría, entre los colaboradores de la empresa. Por lo que se considera que cuentan con una satisfacción laboral entre los nivel alto y muy alto.

Para las características demográficas, se obtuvo que el género, el estado civil y la escolaridad alcanzada no es un factor que influya en los niveles de satisfacción laboral de los colaboradores, mientras que la antigüedad laboral si influye, entre más años tienen los colaboradores, más concentración en los niveles positivos de satisfacción se refleja. Sin embargo, es conveniente enfatizar que dado que el estudio se centra en una variable psicológica no es posible extrapolar los resultados a otras organizaciones. 
Los estudios de la satisfacción laboral siguen diversas líneas de investigación y han sido relacionadas con diferentes variables organizacionales. Su importancia radica en ser una variable dependiente del comportamiento organizacional que hoy en día es valorada en las diferentes organizaciones. Determinar el nivel de satisfacción laboral favorece que se tomen decisiones orientadas a alienar los intereses de los empleados con los objetivos de la organización, estos es que los adopten como suyos y los ejecuten, al sentirse satisfechos y parte de organización.

Los resultados de la investigación reflejan que la satisfacción laboral es condicionante del comportamiento de los individuos al interior de las organizaciones; la revisión de la literatura la presenta como una variable psicológica que modela la actitud laboral ya que serán las percepciones las que configuren la realidad organizacional. En consecuencia se corrobora la relación positiva con el logro de los objetivos organizacionales coincidiendo con la perspectiva teórica de diversos estudios empíricos (Arnold, Robertson y Cooper, 1991; Beer, 1964; Bravo, 1992; Griffin y Bateman, 1986; Harpaz, 1983; Peiró, 1984; Salancik y Pfeffer, 1977).

La afirmación anterior no admite diferencias entre el giro, tamaño o naturaleza de las organizaciones el alcance de los objetivos dependerá principalmente de los individuos. Por lo que es necesario prestar la debida atención -incluso desde el proceso de selección de personal- y gestionar estas dimensiones para elevar su nivel de satisfacción laboral incidiendo en el estado de ánimo de cada colaborador, propiciando se reduzca la brecha entre los intereses individuales y organizacionales.

Si bien es ciertos existen diversos estudios en torno a la determinación de la satisfacción laboral en las organizaciones, la riqueza de cada investigación es factible de apreciar al permitir aproximarse a la comprensión de las dinámicas complejas que se gestan al interior de las organizaciones. 


\section{FINANCIAMIENTO}

No monetario.

\section{AGRADECIMIENTO}

A la Universidad Juárez Autónoma de Tabasco; por motivar el desarrollo de la Investigación.

\section{REFERENCIAS CONSULTADAS}

Adrián, T., Buboltz, W. C., Winkelspecht, C. S. (2004). Características del trabajo y personalidad como predictores de satisfacción laboral. [Job characteristics and personality as predictors of job satisfaction]. Revista Internacional de Análisis Organizacional, 12. Núm. 2, pp. 205-219. Obtenido de https://n9.cl/o2bn

Atalaya, M. C. (1999). Satisfacción laboral y productividad. [Job satisfaction and productivity]. Revista de Psicología. (3) Obtenido de https://n9.cl/5800

Blanco. N. E. (2015). Factores de incidencia en la satisfacción laboral de los individuos en las organizaciones (Diplomado). [Factors of incidence in job satisfaction of individuals in organizations]. Universidad militar nueva granada. Colombia. Obtenido de https://n9.cl/h79p

Boluarte A. (2014). Propiedades psicométricas de la Escala de satisfacción laboral de Warr, Cook y Wall. [Psychometric properties of the job satisfaction scale of Warr, Cook and Wall]. Investigación original, (25) pp. 80-84. https://doi.org/10.20453/rmh.v25i2.249

Chiang, M., Méndez, G. y Sánchez, G. (2010). Cómo influye la satisfacción laboral sobre el desempeño: Caso empresa de Retail. [Job Satisfaction Influence Performance: Business Retal]. Theoria, vol. 19, (2), pp. 21-36. Obtenido de https://n9.cl/nisxm

Chiang, M., y San Martín N. (2015). Análisis de la Satisfacción y el Desempeño Laboral en los Funcionarios de la Municipalidad de Talcahuano. [Analysis of work satisfaction and performance in public employees of Talcahuano City Hall]. Ciencia $y$ Trabajo. vol 17 (54) pp. 159-165. https://doi.org/10.4067/S0718$\underline{24492015000300001}$ 


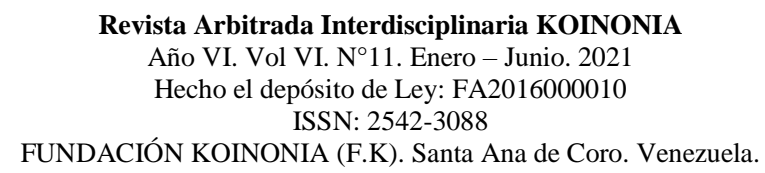

Isabel Solís-Magaña; Fabiola de Jesús Mapén-Franco; Wilver Méndez-Magaña

Hernández, S. R., Fernández, C. C. y Baptista, L. P. (2014). Metodología de la investigación. [Investigation methodology]. México. Ed. Mc Graw Hill.

Herrera, J. M., y Sánchez, I. (2012). Satisfacción Laboral en Trabajadores del IPN un Análisis Estructural. [Job satisfaction in workers of the NPI. a structural analysis]. Ra Ximhai, vol 8, (2), pp. 217-232. https://doi.org/10.35197/rx.08.02.2012.02.jh

Juárez, A. S. (2012). Clima Organizacional y Satisfacción Laboral. [Organizational Climate and Job Satisfaction]. Revista Médica del Instituto Mexicano del Seguro Social, vol. 50, (3), pp. 307-314 Obtenido de: https://n9.cl/8x8p9

Mapén, F. Labor satisfaction and organizational commitment: a correlational study in Mexico. Revista Inclusiones Vol: 7 num 2 (2020): 598-618.

Mañas, M. A., Salvador, C., Boada, J., González, E., Agulló, E. (2007). La satisfacción y el bienestar psicológico como antecedentes del compromiso organizacional. [Satisfaction and psychological well-being as antecedents of organizational commitment]. Psicothema, vol. 19, (3), pp. 395-400. Obtenido de https://n9.cl/11thc

Meliá, J. L. y Peiró J. M. (1998). Cuestionarios de Satisfacción Laboral. Psicología de la Seguridad Laboral. [Job Satisfaction Questionnaires. Psychology of Occupational Safety]. Psicología de la seguridad laboral. Obtenido de https://n9.cl/f8hsx

Meliá, J. L. y Peiró J. M. (1998). La medida de la Satisfacción Laboral en contextos organizacionales: el cuestionario de satisfacción S20/23. [The measurement of job satisfaction in organizational settings: The S20/23 Job Satisfaction Questionnaire]. Psicologemas, 5, 59-74. Obtenido de: https://n9.cl/ait1

Pérez-Bilbao J, Fidalgo M. Satisfacción laboral: general escala de satisfacción laboral. [Job satisfaction: general job satisfaction scale]. Barcelona: Centro Nacional de Condiciones de Trabajo; 1995. https://n9.cl/wkx8

Pilar, A. M. (2008). Estudio comparativo de la satisfacción laboral en el personal de administración. [A comparative study of work satisfaction among public servants]. Revista de Psicología del Trabajo y de las Organizaciones. Vol. 24, (1), pp. 25-40. https://doi.org/10.4321/s1576-59622008000100002

Ramírez, V. y Mapén, F. (2019). Evaluación del Compromiso Organizacional de Servidores Públicos en México. [Evaluation of the Organizational Commitment of Public Officials in Mexico]. Revista Arbitrada Interdisciplinaria KOINONIA. IV (2), 166-189 http://dx.doi.org/10.35381/r.k.v4i8.263 
Revista Arbitrada Interdisciplinaria KOINONIA

Año VI. Vol VI. N 11 . Enero - Junio. 2021

Hecho el depósito de Ley: FA2016000010

ISSN: 2542-3088

FUNDACIÓN KOINONIA (F.K). Santa Ana de Coro. Venezuela.

Isabel Solís-Magaña; Fabiola de Jesús Mapén-Franco; Wilver Méndez-Magaña

Rodríguez, A., Paz, M., Lizana, J. Cornejo, F. (2011). Clima y satisfacción laboral como predictores del desempeño: en una organización estatal chilena. [Organizational climate and job satisfaction as performance predictors in a chilean public organization] Salud y Sociedad. vol. 2, (2). pp. 219-234. Obtenido de: https://n9.cl/pkvo

Salgado, J., Remeseiro, C., Iglesias, M. (1996). Clima organizacional y satisfacción laboral en una PYME. [Organizational climate and job satisfaction in an SME]. Psicothema. vol. 8, (2), pp. 329-335. Obtenido de: http://www.psicothema.com/pdf/31.pdf

Sánchez, M. G. y García, M. L. E. (2017). Satisfacción Laboral en los Entornos de Trabajo. Una exploración cualitativa para su estudio. [Job satisfaction in work settings. A qualitative approach for study]. Scientia et Technica, 22, (2), p. 161166. Obtenido de: $\underline{\text { https://n9.cl/gzdb }}$

Tejero, C. A., y Fernández, M. J. (2009). Medición de la Satisfacción Laboral en la Dirección Escolar Relieve. [Measurement of job satisfaction in school administrators]. Revista Electrónica de Investigación y Evaluación Educativa, 15, (2). Obtenido de: https://n9.cl/9ozlx

C2021 por los autores. Este artículo es de acceso abierto y distribuido según los términos y condiciones de la licencia Creative Commons Atribución-NoComercial-Compartirlgual 4.0 Internacional (CC BY-NC-SA 4.0)

(https://creativecommons.org/licenses/by-nc-sa/4.0/). 